\title{
X-ray absorption spectroscopy study of metal-organic frameworks functionalized by Pd: Formation and growth of Pd nanoparticles
}

\author{
E. Kamyshova, ${ }^{1}$ A. Skorynina, ${ }^{2}$ A. Lazzarini, ${ }^{2}$ A. Bugaev ${ }^{1}$, U. Olsbye, ${ }^{2}$ A. Soldatov ${ }^{1}$ \\ ${ }^{1}$ Southern Federal University, Rostov-on-Don, Russia, \\ ${ }^{2}$ University of Oslo, Norway \\ kamyshova.liza@gmail.com
}

UiO-66/67/68 metal-organic frameworks (MOFs) show an incredible thermal and chemical stability, which makes them promising materials for catalysis. Functionalization has played an important role in enhancing the material potentialities, by insertion of other metals in the inorganic cornerstones and by functionalization of linkers by additional metals.

This research is aimed to investigate the structure and catalytic properties of series of new UiO-67 metal-organic frameworks functionalized with palladium by in situ and operando X-ray absorption spectroscopy (XAS). The main goals were to determine (i) the key steps of formation of the nanoparticles in UiO-67 pores, (ii) prove the stability of materials upon activation and reaction conditions, and (iii) obtain the structure-reactivity relationships during catalytic hydrogenation of carbon dioxide.

The synthesized materials were studied at the at BM31 beamline of ESRF (Grenoble, France) by simultaneous Pd K-edge XAS and Xray diffraction (XRD), while the output of the mixture was analysed by online mass spectrometer. XAS was used as the main experimental technique, because of its sensitivity to the local atomic environment around palladium atoms. At the same time, XRD confirmed the stability of UiO-67 crystal structure during the formation of catalytically active species [1].

The samples were activated in situ by heating in a flow of $\mathrm{H} 2$ and $\mathrm{He}$ from room temperature to $300{ }^{\circ} \mathrm{C}$ and left at this temperature for $30 \mathrm{~min}$ to allow the formation of nanoparticles. The activated material was cooled down to $240{ }^{\circ} \mathrm{C}$, and exposed to a reaction mixture (7.5, 2.5, and $10 \mathrm{~mL} / \mathrm{min}$ of $\mathrm{H}_{2}, \mathrm{CO}_{2}$, and $\mathrm{He}$, respectively). The reaction was run at 240,200 , and $170{ }^{\circ} \mathrm{C}$ under total pressure of 1 and 8 bar, for $2 \mathrm{~h}$ under each of the above conditions.

Under reaction conditions, interatomic distances (RPd-Pd) systematically increase for all samples, being larger for lower temperatures and high pressures. After cooling down in reaction mixture, the values of RPd-Pd $\sim 2.81 \AA$ are observed, which are close to that reported for palladium hydrides [2]. However, flushing with helium, gives RPd-Pd $\sim 2.77 \AA$ which is considerably higher than in metallic palladium. This indicates that apart from palladium hydride, an additional phase is present in the samples. The coordination numbers are close to 10 corresponding to the particle size of $2.6 \mathrm{~nm}$ [3]. Within the experimental error, the values are stable and do change during reaction. At the same time, XRD shows a response of UiO-67 crystal structure by increasing the cell parameters with increasing pressure and decreasing temperature, indicative of the adsorption of reactive molecules in MOF.

Summarizing, we showed that under reaction conditions a mixture of palladium hydride and carbide phases is formed during $\mathrm{CO} 2$ hydrogenation in Pd nanoparticles confined inside the pores of UiO-67. The hydride phase is stronger at high pressure and low temperatures, but is removed upon flushing in $\mathrm{He}$, while the carbide one is stable even after flushing.

[1] - Bugaev A. L., Guda A. A., Lomachenko K. A., et al. (2018). Faraday Discuss. 208, 287

[2] - Bugaev A. L., Guda A. A., Lomachenko K. A., et al. (2017) J. Phys. Chem. C 121, 18202

[3] - Kamyshova E. G., et al. Radiat. Phys. Chem. in press, doi: 10.1016/j.radphyschem.2019.02.003.

Keywords: EXAFS; XRD; Pd; catalyst; metal-organic framework; $\mathrm{CO}_{2}$

This research was supported by the Russian Science Foundation, project № 20-43-01015. 